\title{
Applications of Microscopy and Microanalysis in US FDA Forensic Cases
}

\author{
S. Frank Platek, John B. Crowe, Nicola Ranieri and Mark Witkowski \\ US Food \& Drug Administration - Forensic Chemistry Center, 6751 Steger Drive, \\ Cincinnati, Ohio 45237
}

The US Food and Drug Administration (FDA) has regulatory authority over most foods, beverages, pharmaceuticals, cosmetics and medical devices. ${ }^{1}$. Following the Tylenol Tamperings of $1982^{2}$, congressional legislation enacted the Federal AntiTampering Act in 1983 making all actions associated with product tamperings a criminal act. ${ }^{3}$ Seven years later the FDA established the Forensic Chemistry Center (FCC) as a specialized laboratory for the analysis and research of product tampering, counterfeiting, fraud and in the post 9-11 environment, counter-terrorism of FDAregulated products. The FCC's Trace Examination Section uses a variety of instrument and microscopic applications including stereoscopic (SLM), polarized (PLM) and scanning electron (SEM) microscopy along with infrared (FT-IR) and Raman (Raman) spectroscopy and image analysis (IA) in criminal cases and related research. The following are case studies which illustrate the use of these instrumental techniques. The first example involves the examination of a can of infant formula submitted as part of a product tampering case. This case involved a deliberate extortion threat to a grocery store which included the placement of a tampered can of liquid infant formula on the store shelf. The suspect can, marked with an "X" as described by the caller, was recovered from the store and found to have a hole in the can body beneath the re-glued label (Figure 1). SLM analysis of the hole showed it had been drilled and FT-IR analysis of material removed from the hole was found to be consistent with a cyanoacrylate type adhesive. SEM/EDX analysis of the can body, metallic fragments from inside the can and a similar metallic fragment recovered from the suspect's drill bit were indistinguishable from each other. Subsequent chemical analysis of the liquid contents of the suspect can determined high levels of methanol.

In the spring of 2007, a large number of pet deaths attributed primarily to renal failure were associated with contaminated pet foods. Investigations eventually focused on wheat gluten and rice protein concentrates used in the manufacture of the pet foods. The wheat glutens and rice proteins associated with the pet deaths were found to contain melamine and related s-triazine compounds (e.g. cyanuric acid, ammiline, ammelide). The melamine and related s-triazines all containing nitrogen and were used as a means to mimic high protein levels. SLM analysis found suspect particles (Figure 2a) in bulk rice protein samples which were not observed in control rice protein samples. Subsequently an FT- IR analysis found the suspect particles to be consistent with melamine (Figure 2b). Additional experimental work and analysis using PLM, FT-IR and Raman determined that melamine when combined with cyanuric acid produced small, needle-like melamine cyanurate crystals (Figure 2c). Examination of the crystals observed in animal kidney cross sections by PLM and Raman spectroscopy confirmed that these crystal formations were the results of the melamine cyanurate.

An incident in Washington State involving dairy cattle with unexplainable brown sores, skin lesions and ultimately the death of a few cows attracted the attention of multiple government agencies. Skin samples, ears tags and other items from the suspect dairy were analyzed by PLM microchemical analyses (Figure 3), FT-IR, Raman and SEM/ EDS. PLM, microchemical analysis and SEM/EDS-found chromium in the brown residues. FT-IR and Raman spectroscopic analysis found the brown residues to be consistent with a chromic oxide. The lesions were attributed to metal toxicity from a container leaking chromic acid from the loft onto cows in the dairy barn. 
SLM is a primary forensic analysis instrument and coupled with image capture and image analysis software is used for unique applications at the FCC. The analysis of suspect counterfeit pharmaceutical tablets is one application where image analysis (IA) can be used to compare suspect counterfeit and authentic pharmaceuticals. Image capture and analysis of the suspect tablet debossing when overlaid with the authentic tablet image can demonstrate differences between the tablets (Figure 4). IA along with other physical and chemical analysis allows for a complete comparison between suspect counterfeit and authentic pharmaceutical products.

In a research study, SLM, PLM and SEM were used successfully to determine if the needle from a hypodermic syringe had been used to produce punctures in plastic bottles and rubber pharmaceutical vial stoppers. The results of the study concluded that all three techniques (PLM, SLM and SEM) could be used to characterize and differentiate punctures in plastic bottles. SEM and SLM have shown that syringe needles usually produce a very characteristic shape when inserted directly into plastic bottles ${ }^{4}$ (Figure 5a) and rubber vial stoppers ${ }^{5}$ (Figure 5b). The results of the study have been very useful in the analysis of product containers associated with tampering investigations.

1. Federal Food, Drug and Cosmetic; Title 21, Unites States Code, (amended 1998)

2. K.A. Wolnik, et al, Anal Chem (1984); 56(3):466A-474A

3. Federal Anti-Tampering Act; Title 18, United States Code, Section 1365 1983)

4. S.F. Platek et al, SCANNING (2000); 22(2): 58-59

5. S.F. Platek et al, J Forensic Sci. (2002); 47(5): 986-992.
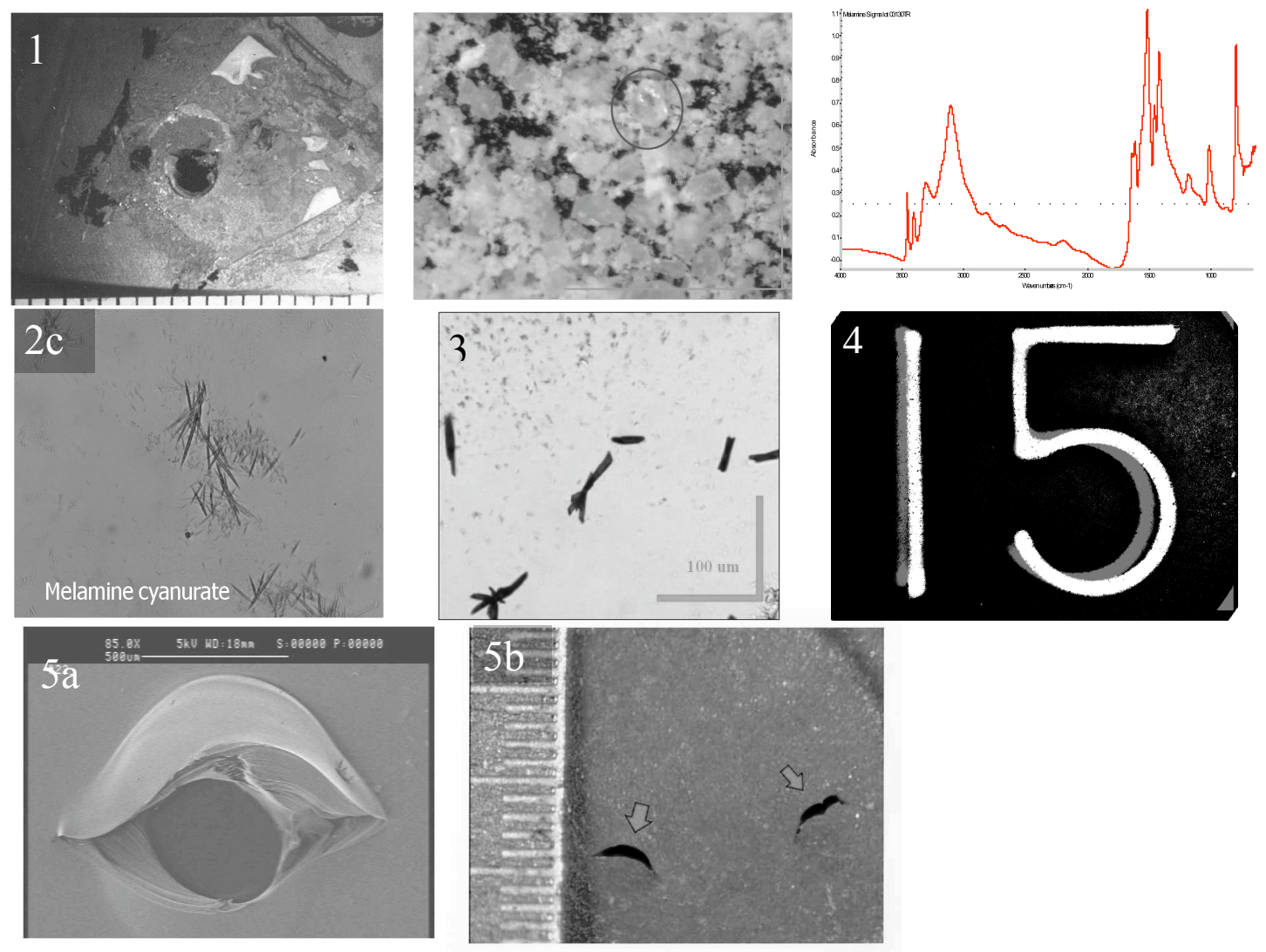

Fig. 1. Drilled hole in infant formula can - site of malicious tampering (SLM).

Fig. 2a. Melamine crystal (circled) in rice protein (SLM).

Fig. 2b. IR spectrum of melamine crystal from rice protein.

Fig. 2c. Melamine cyanurate crystals (PLM).

Fig. 3. Resultant crystals from microchemical test with silver nitrate \& cow ear tag residue (PLM).

Fig. 4. SLM IA of tablet debossing overlaid images showing inconsistencies (suspect grey, known-white).

Fig. 5a. SEM SEI of syringe puncture in plastic bottle showing characteristic piling on one side of puncture.

Fig. 5b. SLM of characteristic syringe punctures in rubber vial stoppers. 